\title{
Fast Atom Bombardment Mass Spectrometry of Mycobacterial Glycopeptidolipid Antigens: Structural Characterization by Charge Remote Fragmentation
}

\author{
L. M. Lopez Marin, D. Promé, M. A. Lanéelle, M. Daffé, and J. C. Promé \\ Centre de Recherche de Biochimie et Génétique Cellulaires CNRS, Toulouse, France
}

\begin{abstract}
Mycobacteria contain species- and type-specific antigens. Among them, glycopeptidolipids are present in medically relevant organisms belonging to Mycobacterium avium or $M$. fortuitum complexes. Fast-atom bombardment mass spectrometry of glycopeptidolipids has proven to be difficult. In this article the cationization method with a metnnitrobenzyl alcohol matrix, doped with sodium iodide, is described for analyzing these molecules. The molecular weight of the intact glycopeptidolipids was successfully determined and, using massanalyzed ion kinetic energy spectrometry, the complete sequences of the peptide and saccharide moieties were elucidated. Moreover, the two structural variants present in these molecules were clearly differentiated. Application of the method showed that the same structural variant occurs in the glycopeptidolipids from two serologically related species of the M. fortuitum complex. (I Am Soc Mass Spectrom 1992, 3, 656-661)
\end{abstract}

$\mathrm{T}$ The cell wall of many opportunistic mycobacteria contains cell surface glycolipids involved in immunological reactions [1]. Among them, a class of glycopeptidolipids (GPL), also called C-mycosides, is frequently described as being composed of a common core to which a saccharide appendage is O-glycosidically linked to the $\beta$-hydroxyl of allo-threonine:

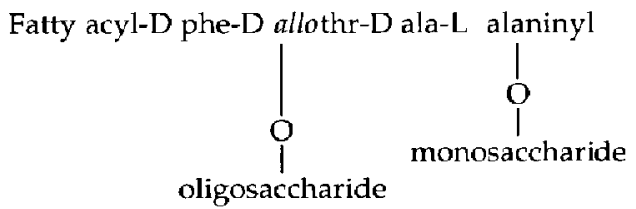

Recently, on the basis of the combined use of mass spectrometry, NMR, and chemical degradation techniques, a new distribution of the saccharide moieties in this glycolipid family has been shown to occur in Mycobacterium fortuitum subsp. peregrinum [2]. In this structural variant a single 6-deoxymonosaccharide is attached to the hydroxyl side of the threonine, and a disaccharide unit is linked to the alaninyl residue.

Fast atom bombardment mass spectrometry (FAB/MS) coupled with mass-analyzed ion kinetic energy (MIKE) analysis applied to the variant molecule

Address reprint requests to J. C. Promé, Centre de Recherche de Biochimie et Génétique Cellulaires CNRS, 118 Route de Narbonne, 31062 Toulouse Cedex, France. has provided important information, because both the molecular weight and the sequence of all constituents were obtained without any derivatization [2].

In this article the method was successfully used to determine the structure of GPLs isolated from another species of the $M$. fortuitum complex, namely $M$. chelonae subsp. chelonae, which shares certain antigens with $M$. fortuitum subsp. peregrinum. Mass spectrometry allowed us to demonstrate the presence of the same structural feature in the GPLs from the two serologically related strains.

Furthermore, to demonstrate its potential value for the discrimination between the two $\mathrm{C}$-mycoside structural variants, FAB-MIKE spectrometry was also applied to the study of a well-known conventional $C$ mycoside from serovar 4 of the $M$. avium complex [3].

\section{Experimental}

Bacteria. M. chelonae subsp. chelonae NCTC 946, M. chelonae subsp. chelonae IP-80-1468, and $M$. avium complex serovar 4 IP-14 0310013 were grown for 2 to 4 weeks on Sauton's medium at $30{ }^{\circ} \mathrm{C}(M$. chelonae strains) and $37^{\circ} \mathrm{C}$ (M. avium).

Extraction and purification of lipids. After growth, cells in a pellicle on the surface were harvested by pouring off the medium, and lipids were extracted by a mixture of chloroform and methanol $(1: 2 \mathrm{v} / \mathrm{v})$. The washed 
crude lipid extracts were fractionated on a Florisil column using a gradient of methanol in chloroform as eluent as previously described [2].

Final purification was achieved by high-performance liquid chromatography (HPLC) in a reverse phase column (Nucleosil C-18) with methanol/chloroform $(75: 25 \mathrm{v} / \mathrm{v})$ as eluent.

Miscellaneous. Mild alkaline deacylation was performed as described by Brennan and Goren [4].

The C-mycosides were hydrolyzed by $1 \mathrm{M}$ $\mathrm{CF}_{3} \mathrm{COOH}$ at $110^{\circ} \mathrm{C}$ for $1 \mathrm{~h}$. The released sugars were analyzed as their trimethylsilyl derivatives by gas chromatography on a capillary fused-silica column (OV-1).

Amino compounds were liberated with $6 \mathrm{M} \mathrm{HCl}$ at $110{ }^{\circ} \mathrm{C}$ overnight and then analyzed on Merck precoated cellulose plates by eluting with 1-butanol/acetic acid/water (4:1:1 v/v/v).

In all cases the previously characterized $\mathrm{C}$-mycoside from $M$. $s p 1217$ [5] was used as a standard.

Mass spectrometry. Mass spectrometry was performed on a ZAB-HS reverse geometry mass spectrometer (VG Analytical, Manchester, UK). FAB spectra were gener- ated by an 8-keV xenon atom beam. Samples were dissolved in methanol/chloroform $(1: 1, v / v)$. On the probe tip $1 \mu \mathrm{L}$ of the solution was mixed with $1 \mu \mathrm{L}$ of metanitrobenzyl alcohol (MNBA) and $1 \mu \mathrm{L}$ of a $10 \%$ solution of sodium iodide in water. Ten scans at 10 $\mathrm{s} /$ decade were accumulated to obtain a spectrum. The resolution of the instrument was set to 1500 . MIKE spectra were measured by electrostatic voltage scanning $(800 \mathrm{eV} / \mathrm{s})$, while keeping the parent ion resolution at 1500 . Between 20 and 40 scans were stored and accumulated for each experiment.

\section{Results and Discussion}

The production of molecular ions by FAB/MS has been described as difficult, and plasma desorption has been preferred for the ionization/desorption of mycobacterial glycopeptidolipids [6]. However, using an MNBA matrix doped with sodium iodide (i.e., the cationization method) intense and long-lasting signals of cationized molecules were produced. In fact, when the samples were only purified by normal phase chromatography, ions at lower mass-to-charge ratio values than expected were mainly observed during the first minutes of the desorption process, and a delay of about 15 minutes was required to obtain abundant

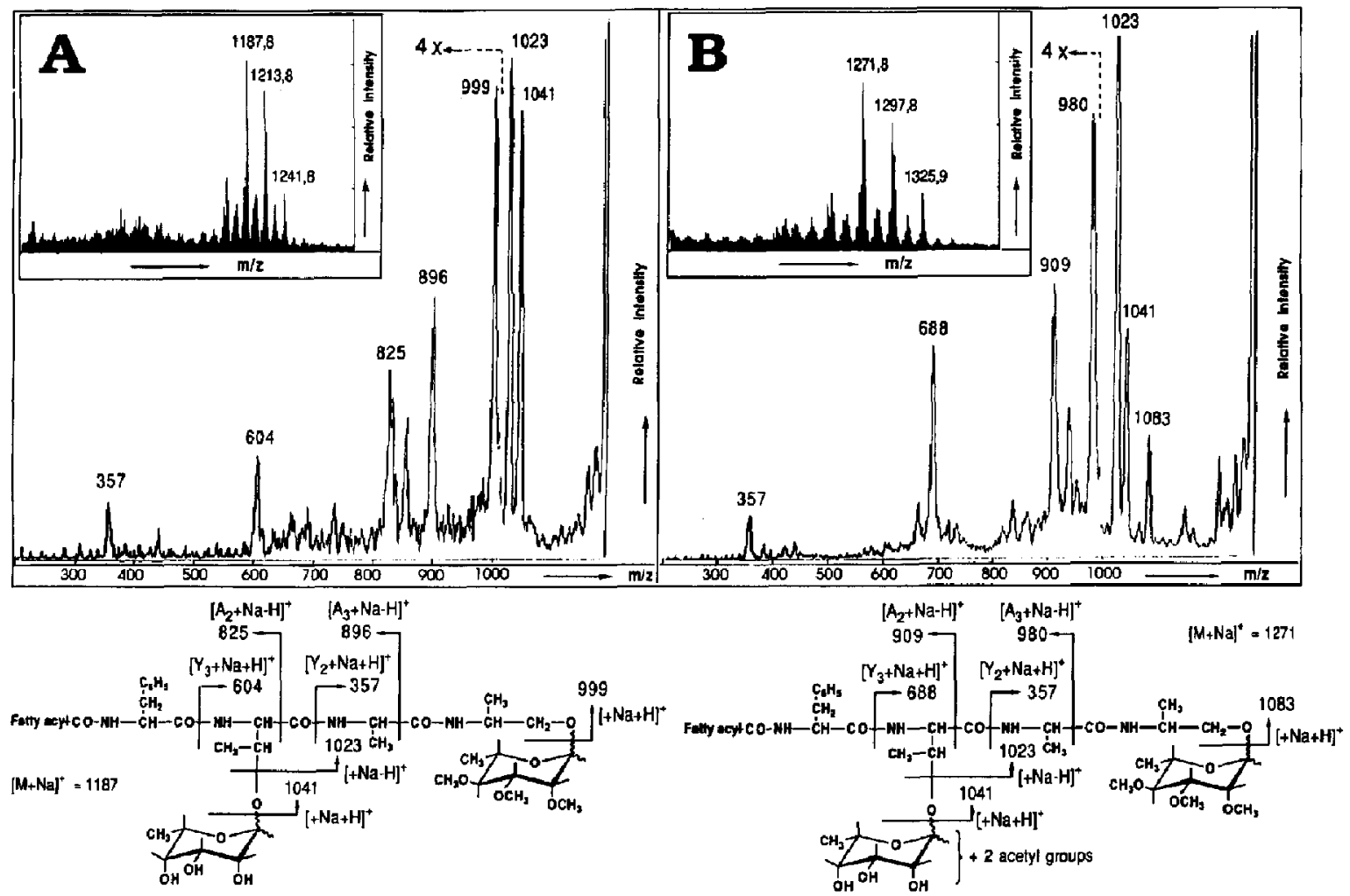

Figure 1. Apolar C-mycoside from $M$. chelonae. (a) FAB (inset) and FAB/MIKE spectra of the deacylated C-mycoside and (b) FAB (inset) and FAB/MIKE spectra of the native C-mycoside, with the corresponding structures. 
cationized molecules from C-mycosides. This indicated that, despite their apparently thorough purification, the samples were still contaminated by lower molecular weight compounds that strongly competed at the beginning of the desorption process. Indeed, the determination of the sugar composition of these samples indicated the presence of glucose in addition to several deoxysugars. As glucose had never been described as a component of C-mycosides, acylated glucose, usually encountered in mycobacteria, was suspected to contaminate the $\mathrm{C}$-mycoside fractions. Thus, a very mild alkaline treatment step was added up to the isolation procedure to remove all ester-linked fatty acids as previously recommended by Brennan and Goren [4]. After this cleaning procedure C-mycosides desorbed almost immediately under the above described FAB conditions. However, the cationized molecular species appeared at lower mass-to-charge ratio value, indicating the presence of $\mathrm{O}$-acyl groups in the intact GPLs, as often occurs in these mycobacterial molecules [1]. Therefore, for mass analysis of the native compounds, a purification step by reverse phase HPLC was alternatively added or, in a simpler way, a time-course study of the FAB desorption process was made by recording only the long-lasting signals. For example, from the $C$-mycoside fractions of $M$. chelonae subsp. chelonae, two compounds were isolated. The most apolar component exhibited three cationized molecules [M + $\mathrm{Na}^{+}$at $m / z 1271.8,1297.8$, and $1325.9( \pm 0.2 \mathrm{Da}$ accuracy), the mass unit difference between them probably corresponding to different chain lengths in their fatty acyl moiety. After the alkaline cleaning procedure, the corresponding cationized species were shifted $84 \mathrm{Da}$ down, indicating the presence of two $O$-acetyl groups (Figure 1, insets).

\section{FAB / MIKE Spectrometry: Sequencing of Individual Components}

All attempts to generate protonated molecular species from C-mycosides abundant enough to allow their tandem mass spectrometry study were unsuccessful. Thus, all the decomposition studies of ionized Cmycosides were carried out on sodium-cationized molecules. As a rule, the main decomposition pathways of cationized molecular species from organic molecules should proceed without charge participation, that is, through charge remote fragmentation processes [7]. As expected, all fragment ions contained the alkali cation. This was easily demonstrated by the examination of the fragmentation spectra from potassium-cationized C-mycosides. All ions were shifted up $16 \mathrm{Da}$.

Metastable ion spectra were preferred to collisionactivated dissociation (CAD) spectra. Although collision processes enhanced the yield of fragment ions, the poor resolution of their analyses induced both a much lower accuracy in the mass assignments (due to the presence of numerous unresolved peaks) and a high background due to a large number of fragment ions differing slightly in mass value. In contrast, the metastable ion spectra (or CAD spectra at very low pressure) were relatively clean, and ionic statistical fluctuations were easily compensated by averaging a large number of spectra. This was easily done because of the long-lasting production of cationized molecules.

\section{Apolar C-Mycoside from $\mathrm{M}$. chelonae}

The apolar C-mycoside from $M$. chelonae exhibited, after alkaline deacetylation, three signals corresponding to sodium cationized molecules at $m / z$ 1187.8, 1213.8, and 1241.8 (see Figure 1a, inset). Chemical acid hydrolysis revealed the presence of both a 6-denxyhexose (6-deoxytalose) and a methylated deoxyhexose (tri-O-methylrhamnose). Amino compounds were identified as the amino acids D-Ala, D-Phe, D-alloThr together with the amino alcohol alaninol.

The MIKE spectrum from the ion at $m / z 1187$ (nominal mass) is presented in Figure 1a. Two large fragments were observed at $m / z 1041$ and 1023. They corresponded to the loss of dcoxytalose both as an anhydro residue $(m / z 1041)$ and as the intact molecule ( $m / z$ 1023). The occurrence of these two types of cleavages at the same saccharide moiety has been shown to be characteristic of sugars directly linked to allo-threonine [2]; thus these fragmentations suggested the 6-deoxytalose residue to be located in that position. In addition, the loss of tri- $O$-methylrhamnose as an anhydro residue only (giving $m / z$ 999) and the absence of any loss of disaccharidyl units suggested that this sugar was linked to the alaninol end of the peptidic backbone.

In the peptidic moiety, cleavages were of the $Y$ and A types, according to the Roepstorff nomenclature [8], with the sodium ion replacing a proton (Figure la). The peak at $m / z 357$ was assigned to the $Y$ cleavage at the threonine-alanine linkage, and the $Y$ type fragmentation between phenylalanine and threonine residues gave a signal at $m / z$ 604; peaks at $m / z 825$ and 896 were assigned to the $A$ type cleavages at the threonine-alanine and the alanine-alaninol linkages, respectively. These fragmentations established the amino acid sequence as being identical to that of $\mathrm{C}$ mycosides. Moreover, the points of attachment of the two sugar residues were clearly determined from these ions: the mass of the sugar residue linked to the allo-threonine side chain was deduced from the mass difference between the ions from the $Y_{3}$ and $Y_{2}$ type cleavages, and the mass of the saccharide linked to the alaninol end was calculated from the mass-to-charge ratio value of the $Y_{2}$ type ion. In this C-mycoside, 6-deoxytalose is located on allo-threonine whereas trio-methylrhamnose is linked to alaninol.

To locate the two acetyl groups the MIKE spectrum of the native molecule (Figure $1 \mathrm{~b}$ ) was compared with that of the deacylated molecule (Figure 1a). The MIKE spectrum from the parent ion at $m / z 1271$ (nominal 


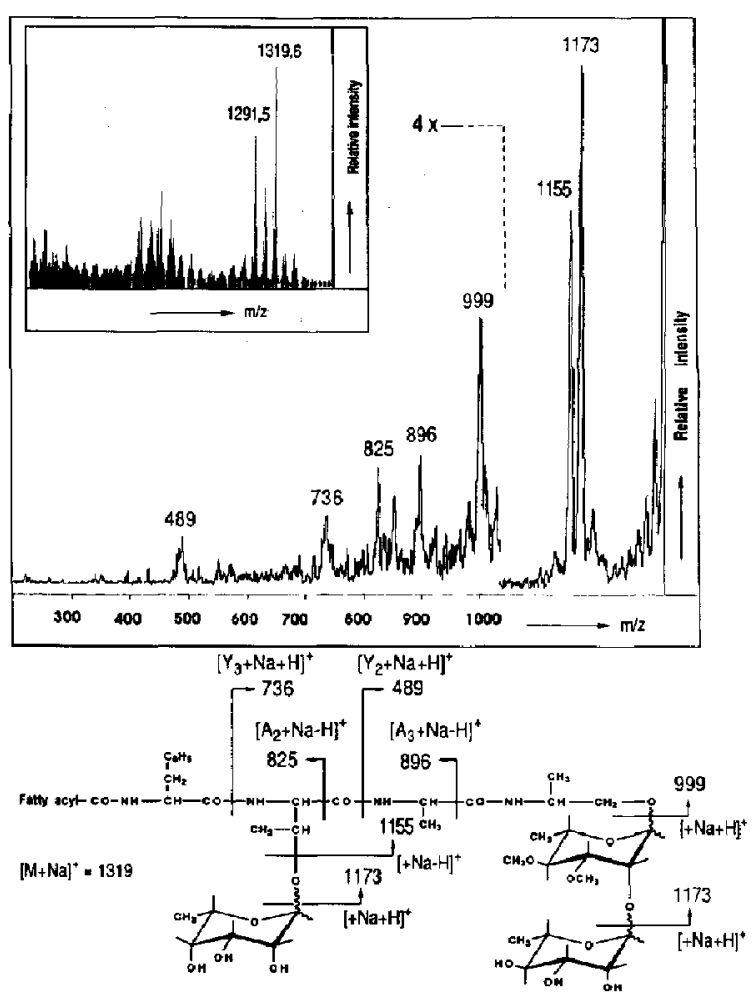

Figure 2. Polar C-mycoside from $M$. chelonae. FAB mass spectrum of the polar deacylated C-mycoside (inset) and FAB /MIKE spectrum and structure of the sodium cationized molecule at $m / z 1319$ (nominal mass). Although rhamnose and 6-deoxytalose are stereoisomers and consequently not differentiated by mass spectrometry, it can be assumed that 6-deoxytalose is linked to alla-threonine, as in the apolar homologue.

mass) showed that the $Y_{2}$ type ion was still observed at $m / z 357$, whereas all the other ions resulting from the peptidic cleavages were shifted up by $84 \mathrm{Da}$. This observation allowed the location of the two acetyl groups on the deoxytalose residue, also confirmed by the fragmentation of the sugar residues: the loss of 6-deoxytalose produced the same ions (at $\mathrm{m} / z 1041$ and 1023) from both the native and the deacylated molecule (Figure 1).

The fragment ion attributions were confirmed by measuring the MIKE spectra of the sodiated homologous molecules at $m / z 1213$ and 1297 (nominal masses; see Figure 1), which showed a mass shift only in the acyl-containing fragment ions.

\section{Polar C-Mycoside from $\mathrm{M}$. chelonae}

The native compound exhibited sodium-containing molecules at $m / z 1375.8,1389.8$, and $1403.8( \pm 0.2)$, which were also shifted down $84 \mathrm{Da}$ after alkaline treatment to $1291.5,1305.6$, and $1319.6( \pm 0.2)$, respectively (Figure 2, inset).

Chemical acid hydrolysis liberated three different sugars, namely 6-deoxytalose, 3.4-di-O-methylrhamnose, and rhamnose.

Figure 2 shows the MIKE spectrum of the sodium cationized major homologue (nominal mass 1319) from the deacylated $C$-mycoside. An intense fragment ion at $m / z 1173$, consistent with the loss of an anhydrodeoxyhexose, was observed. The high intensity of the peak was explained by the superimposed loss of either anhydrodeoxytalose or anhydrorhamnose. The elimination of an intact deoxyhexose molecule, giving an ion at $m / z 1155$, was also observed. Interestingly, the ion at $m / z 999$ was assigned to the elimination of an anhydrodisaccharidyl unit composed of a deoxyhexose plus a di-O-methylrhamnose, so it can be assumed that di-O-methylrhamnose is the sugar directly linked to the peptide core because its loss was not observed. The two saccharide moieties were easily located: the disaccharidyl unit was only lost as an anhydro molecule, whereas the deoxyhexose was eliminated both as an intact and an anhydrosugar, suggesting their positions on the alaninol and on the threonine side chains, respectively. Moreover, the difference of $247 \mathrm{Da}$ between the ions from the $Y_{2}$ and the $Y_{3}$ type fragments of the peptidic backbone confirmed that only a deoxyhexose residue is borne on the allo-threonine side chain. This result was also supported by the mass-to-charge ratio values of the $\left[\mathrm{A}_{2}+\mathrm{N}_{\mathrm{a}}-\mathrm{H}\right]^{+}$and $\left[\mathrm{A}_{3}+\mathrm{Na}-\mathrm{H}\right]^{+}$ ions, which were identical to those observed in the spectrum of the apolar homologue (Figure 1a).

\section{Polar C-Mycoside from the M. avium Complex}

MIKE analysis was also successful for determining the complete sequence of orthodox polar C-mycosides. This is illustrated by the mass spectrometric analysis of the GPL from $M$. avium serovar 4, which has a well-established structure [3]. The FAB spectrum showed several sodium cationized species at the expected mass-tocharge ratio values (Figure 3 , inset). The MIKE spectrum of the major one, at $m / z 1677$ (nominal mass), is shown in Figure 3. The loss of the di-O-methylrhamnose linked to the alaninol residue, as an anhydro moiety, gave an ion at $m / z 1503$. From the cleavages of the glycosidic bonds of the tetrasaccharidic part (giving $m / z 1517,1357,1211$, and 1065), it was possible to characterize the sugar sequence, in complete agreement with the established structure. Other saccharide fragmentations were also present in this molecule: the ion at $m / z 1545$ may be interpreted as a ${ }^{1,5} \mathrm{X}$ ring cleavage [11] of the terminal di-O-methylrhamnose and the weak peak at $m / z 653$ as the detached oligosaccharidic part (C cleavage) associated with the sodium ion [11]. It could be noted that, as expected, the cleavage of the bond between allothreonine and the tetrasaccharide moiety proceeded in two ways: elimination of the anhydrotetrasaccharide (giving $m / z$ 1065) and of the intact tetrasaccharide (producing an abundant ion $18 \mathrm{Da}$ lower at $\mathrm{m} / z$ 1047). 


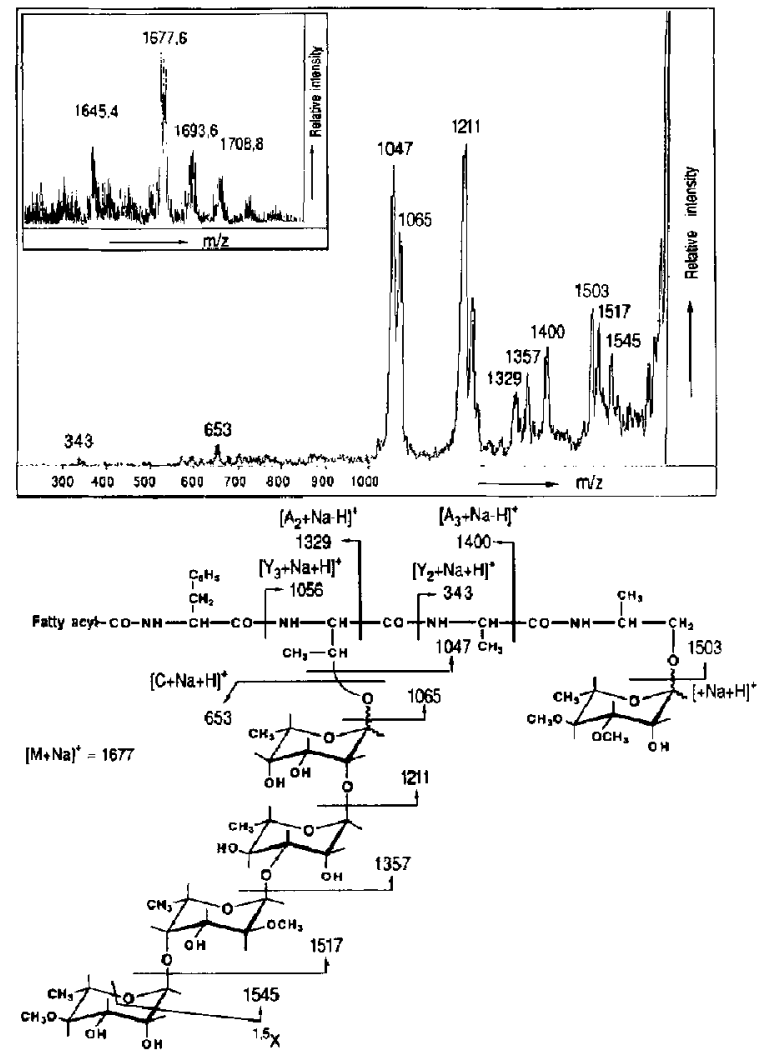

Figure 3. Polar C-mycoside from $M$. avium serovar 4. FAB mass spectrum of the deacylated $C$-mycoside from $M$. avium serovar 4 (inset). FAB/MIKE spectrum of the cationized molecule at $m / z 1677$ (nominal mass), with the corresponding structure.

In the peptidic backbone several cleavages were seen, giving the $\left[\mathrm{A}_{3}+\mathrm{Na}-\mathrm{H}\right]^{+}$and $\left[\mathrm{A}_{2}+\mathrm{Na}-\right.$ $\mathrm{H}^{+}$ions that occurred at $m / z 1400$ and 1329 , respectively. The $\left[\mathrm{Y}_{3}+\mathrm{Na}+\mathrm{H}\right]^{+}$ion, which should occur at $m / z 1056$, could not be seen, as it was probably masked by the two partially resolved abundant peaks centered at $m / z 1047$ and 1065; the $\left[\mathrm{Y}_{2}+\mathrm{Na}+\mathrm{H}\right]^{+}$ion, expected at low mass $(\mathrm{m} / z$ 343), was extremely weak. However, the amino acid sequence was successfully elucidated by the presence of the $A$ type peptidic cleavages.

\section{Fragmentation Pathways}

Fragmentations of gas-phase complexes between alkali metal ions and peptides have been studied by several workers [10-19]. Four types of sequence ions are mainly observed: (1) cleavages through $\mathrm{H}(\mathrm{R}) \mathrm{C}-\mathrm{CO}$ bonds producing the $\mathrm{N}$-terminal $[\mathrm{A}+\mathrm{Cat}-\mathrm{H}]^{+}$ions where hydrogen is transferred from the fragment ion to a neutral leaving group, (2) cleavages through amide bonds associated with a hydrogen transfer from a neutral leaving group to the fragment ion, affording the C-terminal $[\mathrm{Y}+\mathrm{Cat}+\mathrm{H}]^{+}$or $[\mathrm{Y}+\mathrm{Cat}-\mathrm{H}]^{+}$ ions, (3) cleavages through amide bonds with $\mathrm{H}$-transfer from the fragment ion to the neutral leaving group, giving the N-terminal $[\mathrm{B}+\mathrm{Cat}-\mathrm{H}]^{+}$ions, and (4) cleavages through amide bonds with participation of the terminal carboxyl group, giving the N-terminal $\left[\mathrm{B}_{n-1}+\mathrm{Cat}+\mathrm{OH}\right]^{+}$ions.

The mycobacterial GPLs studied here possess neither a free carboxylic group nor a free amino group. The fragments of the peptidic backbone were of the $[\mathrm{A}+\mathrm{Cat}-\mathrm{H}]^{+}$and $[\mathrm{Y}+\mathrm{Cat}+\mathrm{H}]^{+}$types only:

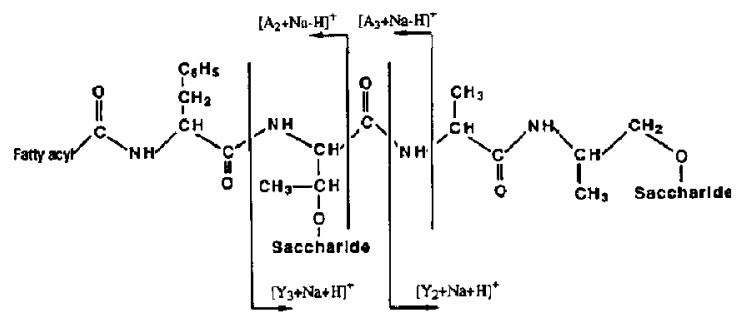

$[\mathrm{A}+\mathrm{Cat}-\mathrm{H}]^{+}$ions were obtained, supporting the mechanism proposed by Teesch et al. [12] in which no free carboxylic group is required, as had been previously reported [11]. Only the $A_{1}$ cleavage-between threonine and phenylalanine-was not observed, as expected for a fragment that does not possess sufficient polarity to bind the alkali metal ion.

$[\mathrm{Y}+\mathrm{Cat}+\mathrm{H}]^{+}$type ions were obtained from cleavages at the alanine-threonine bond, as well as at the threonine-phenylalanine bond. However, the mechanism proposed for the formation of these ions, that is, 1,2-elimination [10], does not explain the absence of both the $\left[Y_{4}+\text { Cat }+H\right]^{+}$and the $\left[Y_{1}+\right.$ Cat $+\mathrm{H}^{+}$ions. This indicated that carbohydrate-containing fragments, although known to be good binding sites for the alkali ions [20], are not the only involved sites in GPLs. If, in the 1,2-elimination, the alkali ion serves only as a spectator ion primarily to carry the charge $[21,22]$, there is again a priori no reason for the nondetection of these ions. Therefore, as also pointed out by Tomer et al. [19], other mechanisms involving the participation of the alkali ion (by increasing the acidity of the transferred hydrogen) can be also envisioned.

At the carbohydrate moieties cleavages occurred, especially at the glycosidic linkages. Most of the fragments were due to the elimination of anhydrosugar residues, as mainly occur in glycoconjugates [9], except for the scission of the threonine-sugar bond in which two mechanisms occur competitively: elimination of anhydro moieties, with hydrogen transfer from the sugar part to the fragment ion, and elimination of the carbohydrate part as free reducing sugar, with a $\mathrm{H}$ transfer from the peptidic backbone to the neutral leaving group. This last process occurred probably because of the presence of an acidic proton ( $\alpha$-proton of the amino acid residue), leading to an easy 1,2- 
elimination [10]. It should be noted that this last mode of cleavage was not present at the alaninol attachment site, as observed with chemically modified GPLs [2], thus allowing an easy differentiation between the two saccharide moieties.

\section{Conclusions}

From the present work and our previous investigation, devoted to the characterization of new C-mycosides from $M$. fortuitum subsp. peregrinum [2], it can be concluded that the formation and the decomposition studies of sodium cationized molecules generated by FAB represent a convenient technique for the structural elucidation of the mycobacterial GPL antigen family (C-mycosides). The method allows their molecular weight determination as well as the complete sequencing of the components. The saccharidic arrangements of the two glycosylated sites can be clearly deduced, both by the examination of the peptidic cleavages and the losses of the sugar residues. Thus, this mass spectrometric method allowed us to discriminate between the two structural variants occurring in $\mathrm{C}$-mycosides. The demonstration of a new saccharide arrangement in two species belonging to the $M$. fortuitum complex [2, and this work] is in agreement with the serological data showing that the species share common antigens [23]. From the present work it should be noted that the structure established herein for the polar $C$-mycoside of $M$. chelonae subsp. chelonae differs from that tentatively proposed by Tsang et al. [23] for a GPL isolated from the same subspecies and having the same glycosyl composition. Such discrepancies exemplify the difficulties encountered in sequencing these complex molecules and encourage the use of FAB /MIKE spectrometry for their structural elucidation.

\section{Acknowledgments}

We are grateful to Drs. V. Lévy-Frébault and $\mathrm{H}$. David for providing $M$. chelonae and $M$. avium strains. L. M. L. M. received a fellowship from the Consejo Nacional de Ciencia y Tecnologia (Mexico). P. Winterton revised the manuscript.

\section{References}

1. Brennan, J. P. In Microbial Lipids, Vol 1; C. Ratledge and S. G. Wilkinson, Eds.; Academic Press: London, 1988; pp 251-278.

2. Lopez Marin, L. M.; Lanéelle, M. A.; Promé, D.; Daffé, M.; Lanéelle, G.; Promé, J. C. Biachemistry 1991, 30, 10536-10542.

3. McNeil, M.; Tsang, A. Y.; Brennan, P. J. J. Biol. Chem. 1987, $262,2630-2635$.

4. Brennan, P. J.; Goren, M. B. J. Biol. Chem. 1979, 254, 4205-4211.

5. Lanéelle, G.; Asselineau, J. Eur. J. Bichem. 1968, 5, 487-491.

6. Jardine, I.; Scanlan, G.; McNeil, M.; Brennan, J. P. Anal. Chem. 1989, 61, 416-422.

7. Adams, J. Mass Spectrom. Reviews 1990, 9, 141-186.

8. Roepstorff, P.; Fohlman, J. Biomed. Mass Spectrom. 1984, 11, 601.

9. Domon, B.; Costello, C. E. Glycoconjugate J. 1988, 5, 397-409.

10. Teesch, L. M.; Adams, J. J. Am. Chem. Soc. 1991, 113, 812-820.

11. Grese, R. P.; Cerny, R. L.; Gross, M. L. J. Am. Chem. Soc. 1989, $111,2835-2842$.

12. Teesch, L. M.; Orlando, R. C.; Adams, J. J. Am. Chem. Soc. $1991,113,3668-3675$.

13. Leary, J. A.; Zhou, Z.; Ogden, Z. A.; Williams, T. D. J. Am. Soc. Mass Spectrom. 1990, 1, 473-480.

14. Leary, J. A.; Williams, T. D.; Bott, G. Rapid Comm. Mass Spectrom. 1989, 3, 192-196.

15. Tang, $X . ;$ Ens, W.; Standing, K. G.; Westmore, J. B. Anal. Chem. 1989, 60, 1791-1799.

16. Reruner, D.; Spiteller, G. Biomed. Environ. Mass Spectrom. 1988, 15, 75-77.

17. Russell, D. H.; McGlohon, E. S,; Mallis, L. M. Anal. Chem. $1988,60,1818-1824$.

18. Grese, R. P.; Gross, M. L. J. Am. Chem. Soc. 1990, 112, $5098-5104$.

19. Tomer, K. B.; Deterding, L. J.; Guenat, C. Biolog. Mass Spectrom. 1991, 20, 121-129.

20. Prome, J. C.; Puzo, G. Org. Mass Spectrom. 1977, 12, 28-32.

21. Röllgen, F. W.; Borchers, F.; Giessmann, V; Levsen, K. Org. Mass Spectrom. 1977, 12, 541-543.

22. Röllgen, F. W.; Borchers, F.; Giessmann, V.; Levsen, K. Org. Mass Spectrom. 1978, 13, 459-461.

23. Tsang, A. Y.; Barr, V. L.; McClatchy, J. K.; Goldberg, M.; Drupa, I.; Brennan, P. J. Int. J. Syst. Bacteriol. 1984, 34, 35-44. 